\title{
GEOPHYSICAL RESEARCH AND PROGRESS IN EXPLORATION*
}

\section{J. E. WHITE, $\dagger$ AND FRANK PRESS $\ddagger$}

\begin{abstract}
This is the fourth in a series of review papers dealing with technological developments in exploration and with geophysical research at universities and industrial laboratories.

Among the developments in exploration, the use of reproducible recording of seismic records continues to grow, with a trend toward digitizing reflection data for further computation. Refined digital methods have been developed for computation of synthetic seismograms. Well-logging developments have included the appearance of a resistivity logger capable of being pumped down the drill pipe; the availability of more tools for logging seismic velocity, accompanied by laboratory investigations of the manner in which seismic velocity is affected by porosity, fluid content, and other factors; commercial use of the scattered-gamma ray density tool; and further interest in gamma ray spectral logs. Remarkable advances have been made in the techniques of gravity measurement aboard surface vessels and aircraft, although the results are not directly applicable to prospecting. The proton precession magnetometer is being used in commercial air-borne surveys, but the rubidium vapor magnetometer is not available as an exploration tool. The development of a Doppler-positioned navigation method has greatly facilitated air-borne surveys. A ship-borne seep-detector has been outfitted for exploration in water-covered areas.

A review is presented of research on all phases of geophysics at academic institutions in the United States, including laboratory experiments, field measurements, and theory. The review also gives partial coverage of geophysical research at European laboratories, including a discussion of Soviet research as gauged by recent visits to a number of research laboratories in the Soviet Union.
\end{abstract}

\section{INTRODUCTION}

At the Annual Meeting of the SEG in Denver in 1955, the SEG Research Committee sponsored a paper which reviewed significant developments in exploration technology during the previous year. In 1956 the second paper in this series was given, covering exploration technology but featuring a review of research projects at universities and other non-commercial institutions relating to exploration geophysics. The third paper sponsored by the Research Committee, presented at the Annual Meeting in 1957, reviewed recent technological developments in Europe, as well as developments in exploration methods in the United States. Since no paper was given in 1958, the present paper will cover a two-year period, attempting to describe briefly the main developments in exploration methods within the United States; to review geophysical research at academic laboratories within the United States; to mention some research topics currently being investigated at European laboratories, without any claims as to completeness of coverage; and to pass on some impressions gained during recent visits to Soviet geophysical research laboratories.

* Presented at twenty ninth International Meeting, November 9, 1959. Manuscript received by the Editor October 8, 1959.

$\dagger$ The Ohio Oil Company, Littleton, Colorado.

$\ddagger$ California Institute of Technology, Pasadena, California. 


\section{DEVELOPMENTS IN EXPLORATION TECHNOLOGY}

\section{Seismic Prospecting}

Techniques for reproducible recording of seismic records, which have been described in earlier review articles of this series, have undergone further development by many manufacturers of seismic equipment and have gained such widespread acceptance that the use of magnetic recording may well be considered standard. Several equipments exist which will reliably produce a time-corrected record-section, in wiggle-trace, variable area, or variable density presentation, usually in a central office playback facility. Miniaturization of recording and play-back equipment is proceeding rapidly, making it easier to carry out the complete record analysis in remote field locations.

Digital computers offer an attractive means of making routine computations on seismic data, as evidenced by activity among several research groups during this period. Starting with reflection times read from records in the conventional way and punched into cards, one group has used a digital computer to compute true depths for as many as 13 reflection events, with automatic plotting of the depth points. Velocity functions may be changed readily, and cross-sections with some chosen reflection as a datum can be prepared easily. The time-consuming operation of picking the reflection times and punching them into cards has been avoided by another research group, who have developed an analog-to-digital converter which senses any selected trough and punches the reflection time automatically. The interpreter indicates on a monitor record the troughs of interest, and then the converter makes the time pick to an accuracy of one millisec from the corresponding magnetic tape record. Still another group has developed the digital computer routines to compute migrated cross-sections from such digital reflection-time data, which are then plotted on a specially designed geophysical plotter. This instrument plots on a $40 \times 40$ inch surface at a rate of approximately one point each $1 \frac{1}{2} \mathrm{sec}$. When plotting seismic cross-sections, it plots a reflection as a line segment of suitable length and inclined at a dip angle controlled by the input. Reflection quality is indicated by variation of the line "weight" and texture. A central symbol is printed at the $x, y$ coordinate point of the reflector. In addition to seismic cross-sections, the plotter is also applicable to mapping problems in which $x$ and $y$ are surface coordinates, and derived or measured parameters may be posted by the digital print.

Digital computers have made possible a major refinement in the application of synthetic seismograms to problems of seismic interpretation. Commercial services now offer synthetic seismograms obtained by electrical filtering of continuous velocity logs, producing a trace equivalent to single reflection at each interface of a wavelet whose shape can be selected. During the last two years, at least three research groups have described digital programs for computing synthetic seismograms from velocity logs including all multiple reflections, lead- 
ing to a number of significant conclusions. Being able to compute the multiple reflections separately and to compare them with the single-reflection contributions is of considerable value. The effect of multiple reflections on the transmitted pulse can also be obtained, and it was shown that passage through a complex section of layers may change the waveform drastically. This means that a reflection from a single isolated interface at depth may be quite complex, simply because the pulse has traversed the complex overlying beds twice, down to the reflector and back to the surface.

No new field techniques for seismic prospecting are to be reported, but perhaps some comments on methods described in earlier review articles are in order. The "Sonoprobe" described earlier has been used further in exploration of watercovered areas, and another group has developed instrumentation for obtaining continuous profiles of shallow sediments in water-covered areas, operating from a single boat at speeds of three to eight knots and portraying beds as much as a $1,000 \mathrm{ft}$ below the bottom. This equipment is known as the "Sparker" from the fact that its sound source is a 10,000 volt spark fired underwater, usually at a repetition rate of two to four sparks per sec. Little information beyond that given in the last review article could be obtained on the seismic method utilizing a frequency-modulated continuous earth vibrator ${ }^{1}$ in place of dynamite, although it is apparently undergoing further development and evaluation. Weight-dropping has gained wider acceptance during this period, there now being 12 domestic crews and two or three foreign crews in operation.

\section{Gravity Measurements}

Undoubtedly the most exciting developments in the techniques of gravity measurement have been the demonstrations of accuracies which can be achieved aboard surface vessels and in airplanes. ${ }^{2}$ The LaCoste and Romberg submarine or surface ship gravity meter has low enough drift and is capable of averaging the 50,000-mg accelerations of a surface ship well enough to permit readings to be taken with a precision of about one mg. Ship navigation then becomes a controlling factor, since a one-mile error in location can give an error of about one $\mathrm{mg}$ and an error in ship speed of one knot can give an error of about seven mg. From a number of cruises, it is concluded that gravity surveys from surface ships can be made to an accuracy of two $\mathrm{mg}$. With the addition of an analog computer to speed up the meter reading process, several runs aboard a $\mathrm{KC}-135$ jet airplane at Edwards Airforce Base and another set of tests aboard a B-17 aircraft indicated an accuracy of about ten $\mathrm{mg}$ limited by the accuracy with which the position of the plane could be determined throughout the flights. The quality of the results is adequate for geodetic purposes and possibly for some special geophysical applications.

${ }^{1}$ Editor's Note: See page 95, this issue.

${ }^{2}$ Editor's Note: See page 181, this issue. 


\section{Magnetic Methods}

The proton precession magnetometer has been used in many field surveys for research purposes, some of them in connection with the International Geophysical Year. One company has tailored its instrumentation to oil prospecting and offers the proton precession magnetometer as one of its family of aerial surveys, reporting very good performance in every way. Among the advantages of this instrument are its light weight, durability, and absolute calibration. Among its disadvantages are the fact that readings cannot be taken much of tener than twice a sec, which may yield an undesirably low density of observations in an aerial survey, and the fact that only the magnitude of the total field is obtained, with no possibility of measuring the individual components of the field separately.

Another principle applicable to the measurement of the earth's magnetic field was described before the SEG in 1958, but its adaptation to geophysical prospecting usage is apparently at a stand-still. This magnetometer depends on the optical detection of magnetic resonance in rubidium vapor. In one version, the output of a photocell, due to polarized light which has traversed the rubidium vapor, is fed back to a coil surrounding the rubidium cell in such a way that the system oscillates at the Larmor precession frequency. Like the proton precession magnetometer, it indicates the magnitude of the total field. It has the advantages of higher sensitivity and of being able to follow rapid fluctuations. If these features should be especially needed, the ruggedness, small size, and low power drain of the rubidium vapor magnetometer mark it as a likely candidate for field use.

\section{Well-Logging}

As a continuation of the trend pointed out in the last review article, the popularity of seismic velocity logs has continued to grow, aided by the improved availability of velocity logging equipment. Instrumental developments include the multi-receiver tool, which permits a choice of the interval over which the velocity is averaged and hence over the resolution. In addition to continued use of velocity logs as an aid to seismic interpretation, both laboratory measurements on cores and empirical field comparisons have shown the velocity $\log$ to be an effective measure of porosity $y^{3}$ in consolidated formations with intergranular porosity. Although no report has been given of a systematic attempt to use the attenuation of the seismic pulse, as well as its transit time, it has been noted that a virtual disappearance of the received signal may occur in highly fractured zones or in unconsolidated formations bearing gas.

The density logging tool which makes use of a suitably collimated gamma-ray source and scintillation detector has reached the stage of commercial availability, although experience with density logs is still somewhat limited. The close connection between rock density and porosity make this tool very attractive for

${ }^{3}$ Editor's Note: See page 250, this issue. 
formation evaluation, especially in cases where the reservoir rocks may contain variable amounts of shale. It is expected that use of this tool will continue to grow.

A very significant development in nuclear well logging is the experimental demonstration that a borehole particle accelerator can indeed be engineered to meet the rigorous requirements of ruggedness, small size, and lower power consumption imposed by the well logging application. This has made- possible thedownhole generation of 14-mev neutrons, suitable for neutron-activation of elements around the well. Detection and spectral analysis of the resulting gammarays offers the possibility of a reasonably complete analysis of the main elements in the rocks around the borehole. From the standpoint of direct indication of oil saturation, oxygen activation looks particularly attractive, since water and all rocks have an abundance of oxygen, whereas oil has none. Although experimental logs have demonstrated the soundness of this approach, much development remains before it can be considered a proven commercial log.

It should be mentioned that an API subcommittee has sponsored a test facility for calibrating nuclear logging tools and is setting out to standardize scales and sensitivities of neutron and gamma-ray logs from the various companies.

One noteworthy instrumental development during this period is an electriclogging tool capable of being pumped down a drill pipe and out through the bit, thus permitting the logging of a freshly drilled section of hole before mud invasion has progressed and without requiring that the drill stem be removed. A magnetic recorder running for 80 minutes records resistivity and SP logs, after which the tool is pulled out on a wire line. This tool has been successfully field-tested, and a manufacturer is preparing to make it available commercially.

Another development in electric logging which has been successfully fieldtested is referred to as "reverse-wetting logging." Laboratory measurements showed that the resistivity of an oil-saturated core is substantiaily increased by the addition of an agent which tends to make the core preferentially oil-wet, whereas the resistivity of a water-saturated core was unaffected by the agent. As a field test, resistivity logs were run in a well, the well was then reamed with a mud containing the reverse-wetting agent, and the resistivity logs were run again. As expected, the oil-bearing zones showed increased resistivity.

\section{Seep Delector}

An effective and inexpensive technique for locating gas seeps ${ }^{4}$ has been developed for use in water-covered areas. As gas bubbles rise to the surface, small amounts of methane are dissolved in the water, and currents spread this methaneenriched water into a long plume. A boat samples the water continuously, operating on a grid laid out at right angles to the current. Gas is broken out of solution and trace amounts of methane are detected by a non-dispersive infrared analyzer.

'Editor's Note: See page 275, this issue. 
The low cost of this method makes it attractive for localizing areas of interest for subsequent coverage by more expensive exploration methods.

\section{Doppler Positioning}

A major advance in the conduct of aerial geophysical surveys is the adaptation to this purpose of navigational systems used by virtually all high speed militaryjet flights and many commercial planes. It makes use of the fact that when a radar signal from the moving plane is reflected back by objects along the ground, the frequency of the returned echo experiences a Doppler shift which depends on the speed of the plane. By sending out radar beams in four directions, the true ground speed is obtained, and computers keep track of the speed and direction, thereby indicating true position of the plane at all times with surprising accuracy. Thus, pre-selected traverses can be run, even over terrain devoid of land marks, and without need of ground stations.

\section{ACADEMIC RESEARCH}

From the large program of geophysical research in the universities, we have selected topics of special interest to readers of GEOPHYsics. Many important fields, such as isotope geology and mining geophysics, have not been covered. This report could not have been completed without the help of our colleagues at the following universities: Harvard, Columbia, M.I.T., U.C.L.A., California, Stanford, Penn. State, Minnesota, British Columbia, Wisconsin, Toronto, Utah, and Caltech.

The major trends in academic research during the past few years have emphasized world-wide field measurements, the use of electronic computers in data reduction and analysis, and the development of improved instrumentation.

Partly due to the stimulus of the International Geophysical Year and partly a reflection of increased interest in geophysics in the universities, the past two years have seen expanded efforts in world-wide field studies. A wide variety of methods were employed including explosion seismology, earthquake seismology (particularly surface waves), gravity observations, air-borne and ship-towed magnetometers, submarine geophysical methods (coring, photography, echosounding, heat flow).

Extensive surveys have been made of the oceans, including remote seas not heretofore reached by modern research ships. About 1,000 deep sea seismic refraction profiles have been made in the past two to three years. In cooperative ventures such as the recent search in the Atlantic Ocean for a possible site for the Mohole, three or four ship refraction profiles having lengths as great as $70 \mathrm{~km}$ were obtained. The deep-sea work is now directed toward more careful measurements to reveal variations in the physical properties of sediments, crust, and mantle from one region to another.

IGY results in Antarctica, based on a combination of explosion and earthquake data, point to the conclusion that the eastern part of Antarctica is conti- 
nental in structure and that the western half consists of shelves, islands, in an icecovered archipelago. Gravity and reflection seismology show average ice thickness greater than had been assumed. Extensive parts of western Antarctica and coastal regions have ice extending to below sea level.

Studies of the continental crust have seen the use of improved apparatus for recording explosions and the development of new techniques to exploit earthquake surface waves more fully. The importance of the intermediate, often masked, crustal layer in the interpretation of structure was appreciated more fully as a result of work in the USSR, and this layer has now been traced in the Great Basin and in the Canadian Rocky Mountain foothills.

The use of phase velocity of surface waves to reveal regional crustal structure is receiving wider application. The striking correlation found between Rayleigh wave phase velocity and gravity anomaly in North America has important implications regarding isostasy.

An increasing use of geophysical methods for the solution of geological and glaciological problems is noted. Explosion seismology and gravity methods have been used in reconnaissance explorations of geological basins, and in delineating the thickness of glaciers.

Instrumental seismology emphasized further development of long period seismograph systems. Long period pendulums $(30-300 \mathrm{sec})$ and galvanometers $(500 \mathrm{sec})$ were designed. Special transducers, networks and notch filters were used with excellent results. These new instruments, by extending the seismic spectrum, provide data useful in obtaining the properties of the outer layers of the mantle and crust as well as information on the mechanism of the focus.

The analysis of records from these instruments and their theoretical interpretation has yielded exciting results. It was demonstrated from surface wave data that the Gutenberg low velocity zone in the mantle is a world-wide phenomenon. The phase velocity method of exploration was extended to make possible the analysis of crustal structure variations across an entire continent and a significant correlation was found between phase velocity and gravity anomaly.

Surface waves from earthquakes and large explosions continue to be a fruitful field for research. Higher mode propagation has been discovered and analyzed and the structure of the continents, Antarctica, and the deep-sea floor has been scrutinized by these techniques. Advances in the theory of these waves were made and exploited, and electronic digital computers contributed much to these efforts.

Nuclear explosions have provided amplitude and travel time data of seismic body waves which will make possible the delineation in finer detail of the internal constitution of the earth.

Ultrasonic model seismology has progressed to the point where two-dimensional models with continuous variations in density and velocity may be conveniently fabricated.

The need to establish on a firm mathematical foundation the theory of diffrac- 
tion and scattering of elastic waves was recognized and much progress was made. Radiation from different types of sources, scattering of impulsive waves from spheres, cylinders, transmission at corners, and effects of topographic irregularities are some of the problems which have been attacked. Some new mathematical techniques and extensions of old methods were employed.

The report of the Panel on Seismic Improvement (Berkner Panel) has done much to stimulate research in mathematical methods of operating on seismograms to reveal the properties of the source and the propagating medium. It may be recalled that this panel was originally appointed by the President's scientific adviser, Dr. Killian, to study the possibility of improving seismic methods of identification of nuclear explosions. The panel immediately recognized that a broad program of fundamental research was needed. Although this program is now getting underway, preliminary results have shown that information and communication theory when coupled with good understanding of the physics of wave propagation can lead to important progress.

Developments in marine gravimeters have reached the point where important data from these instruments are already being obtained. The Worzel adaptation of the Graf Sea Gravimeter completed a successful program of observations on an 1,800 ton fin-stabilized vessel equipped with a stable platform. Results for the LaCoste marine gravimeter were described earlier.

Reduction of gravity observations and interpretation of anomalies were programmed for electronic digital computers.

Magnetic anomalies revealed in marine surveys have shown interesting patterns for which the tectonic significance has yet to be found. The possibility that displacements along faults may be measured from anomaly patterns has been demonstrated. Paleo-magnetic studies are in the stage where many measurements with wide geographic distribution are needed and are being obtained. Interest is growing in low frequency $(.01-100 \mathrm{cps})$ magnetic fluctuations and these are being measured with instruments suitable for correlation studies over large distances. Research in the use of magneto-telluric methods of exploration is continuing. Particular emphasis seems to be on field testing.

\section{RESEARCH IN THE SOVIET UNION}

We could not hope to cover the large geophysical research program in the USSR adequately. What follows is compiled mostly from the reports of Drs. Yost and Press on their recent trips. It is not an evaluation, but a fragmentary review of selected fields in which original work is being done of interest to readers of GEOPHYSICS.

There is much emphasis on the use of the dynamic characteristics of elastic waves to obtain more information about the sub-surface than time data alone will provide. For example, the absorption coefficients of refraction arrivals are determined and interpreted to distinguish between granitic basement and gneissic basement. The use of absorption coefficients to distinguish between high velocity 
sedimentary layers and basement is also being studied. Attempts are being made to relate variations in absorption coefficient to variations in porosity. Absorption coefficients as determined in acoustic well-logging devices is an additional parameter that is being used. The acoustic logging tool consists of a bank of three equally-spaced receivers with a transmitter on either side. The device is being instrumented for uphole recording of five parameters: compressional velocity, shear velocity, absorption coefficient for compressional waves, frequency, and wave length of the recorded signal.

Spectrum analyses of refraction and reflection arrivals in the range $20-400$ cps are being used for diagnostic purposes, such as determining layer thickness. Theoretical studies and experimental work on the variation of amplitude of refraction arrivals as a function of velocity contrast is being carried on. Field data were cited in support of the theoretical result that the lower the velocity contrast the greater the refraction amplitude. Research is being conducted on multiple refractions and on composite body waves such as PPS or PS. These waves are distinguished by their spectrum as well as the orbital motion at the surface. This work is being carried out in the exploration frequency range for possible application as a field tool. Shear wave reflections at depths of about 2,000 meters were obtained from a dynamite blast.

Correlation refraction shooting is receiving much emphasis. This method is used in exploration for petroleum as well as in studies of the earth's crust. These studies use long refraction profiles whereby a given wave group can be traced over large distances. We were told of one profile having a length of 200 kilometers with an observation at every 200 meters. Use is made of amplitude changes and apparent velocity for diagnostic purposes. This technique has been particularly effective in delineating velocity changes within the crust. The field procedure is to lay down a spread of approximately 10 to 60 groups of geophones, the group separation being 200 meters. Each group consists of four geophones, separated anywhere from 8-15 meters, depending upon the local background noise conditions. Charges of less than $1,000 \mathrm{lbs}$ of TNT are detonated in lakes and by repeated shooting, a profile of 300 or 400 kilometers in length is achieved. The geophones have a natural frequency of nine cps and the overall amplification of the system is ten million in the frequency range 6-9 cps. Radio communication is maintained between the shotpoint and the recording position so that the explosion is delayed if local wind noise prohibits high sensitivity operation of the detectors. The operations are carried out usually in the middle of the night when wind noise is at a minimum, and in regions remote from industrial sites. Thus by putting great effort in their field procedure, the Russians have been able to minimize the effort required at the shotpoint. It is claimed that refraction arrivals from the crustal layers as well as from the Mohorovicic discontinuity can usually be correlated over large distances. The very interesting result is reported that compensation can occur at the level of the Conrad dis- 
continuity as well as at the Mohorovicic discontinuity, and that the complex behavior of these interfaces is reflected in the gravity picture.

Soviet seismologists have been interested in the use of high frequencies in exploration seismology for some years now. They have been working with frequencies as high as $500 \mathrm{cps}$. At these frequencies they feel they can do mapping to a depth of 100 meters. In the frequency range $100-200 \mathrm{cps}$, they have mapped to depths of 2,000 meters. The sources are generally dynamite shots of twofive $\mathrm{kg}$, or some large electromagnetic transducers. The shallow work at high frequencies is primarily designed for locating ore bodies. They have been able to resolve satisfactorily ore bodies 20 meters wide at a depth of 200 meters even when the ore body was very thin.

It is surprising that Soviet work in submarine geophysics was begun only a few years ago, and yet the experimental techniques and results are now at a level comparable to those in the United States, where this field of research was pioneered some twenty years ago. Refraction seismology, topographic surveys, magnetic and gravity observations constitute the tools used in the exploration of such important structures as the Okhotsk Sea, the Kuril Islands, and the Kuril trench.

Research in electromagnetic methods of prospecting is being prosecuted. With one instrument, having a variable oscillator covering the frequency range $0.05-300 \mathrm{cps}$, it is claimed that fairly reliable mapping can be achieved to depths of 2,000 meters. Amplitude and phase comparison yield the basic data. Telluric currents and naturally variable magnetic fields are also being employed.

An interesting research program in geodynamics involves field measurements, theoretical studies, and model studies relating to tectonics. This closely coordinated approach to the problems of geodynamics from three different directions is unique. Materials such as photo-elastic gelatins and viscous fluids were used to model tectonic processes. The photo-elastic effect was used to measure the deformation. Studies of uplift and subsequent relaxation, as well as thrusting were underway.

\section{SOME EUROPEAN RESEARCH ACTIVITIES}

The following comments on geophysical research in Europe are based on recent visits by Dr. White to four research laboratories which place a major emphasis on petroleum exploration.

The program of the British Petroleum Research Centre, Chertsey Road, Sunbury-on-Thames, Middlesex, England, includes diverse topics in geophysics and well logging, as parts of a broader program of research in exploration and reservoir engineering.

Seismic research includes both theoretical and experimental attacks on the generation of seismic waves by explosives, with special attention to the motion very near the shot. Experimental field refraction surveys have been carried out 
and the recorded amplitudes and frequencies compared with theoretical solutions. The refraction process has also been studied by means of seismic modelling, in one case utilizing barium titanate transducers in a fluid layer overlying a solid refracting medium. The Centre has a Burroughs E101 digital computer which is often used in the conduct of the theoretical work.

Relevant to the development of instruments for operating seismic crews, the Centre has environmental test chambers, a mechanical vibration table, and a shielded room. A moving van serves as a mobile laboratory for field trials.

Among other geophysical research topics is the development of a borehole gravimeter, which appears to be well along toward conquering the levelling problems, temperature effects, sensitivity requirements, and other rigorous demands to be met by this tool.

Well logging research includes the development of a neutron-gamma ray tool equipped for pulse-height analysis, comparing the output of a channel set to detect the gamma rays from chlorine with the output of a channel which accepts the broad spectrum of neutron-induced gamma rays from all elements. This is aimed, of course, at locating oil-water contacts. This group is also developing a dip-meter which uses 12 "trumpet-log" electrode buttons spaced around a circumference of the tool. These electrodes are sampled sequentially and the output used to intensity-modulate the time base of an oscilloscope, the brightness corresponding to resistivity. The resultant traces are recorded on a film strip which moves as the tool moves in the hole, producing a pattern on which the dip can be measured. If desired, the film can be bent into cylindrical shape, thus presenting a picture of the wall of the hole.

The program of the Royal Dutch Shell Laboratories in Delft includes topics in geophysical research, as well as geology, sedimentology, and geochemistry. After completion of a new building in 1961, this laboratory will handle the complete subjects of exploration and production research, which will include well logging research now being carried out in Amsterdam.

The electronics section has environmental test chambers and equipment for thorough operational check of seismic systems prior to shipment to the field. The function of this group also includes design of equipment, such as a transistorized refraction amplifier.

Two experimental seismic crews operate from this laboratory. Research interests have included refraction interpretation and the generation of seismic waves. A digital program permits the computation of synthetic seismograms without multiples, with multiples at depth but with no surface reflection, or with both multiples and a surface reflection.

The laboratory of Compagnie Générale de Géophysique located at Montrouge, Seine, France has primary responsibility for developing and maintaining equipment for 50 or 75 geophysical crews, conducting training schools for observers and interpreters, and doing research on new instruments and interpretation methods. 
One instrumental development of this group is the "Toran" radiolocation system for geophysical surveys. Two "focal point" transmitters located at known places transmit in the two megacycle band on unmodulated carrier frequencies which differ by about $100 \mathrm{cps}$. A fixed monitor receiver detects this beat frequency accurately and transmits it to a mobile receiver, whose position is to be determined. The mobile receiver also detects the beat frequency between the two focal point transmitters. A comparison of the phase of the beat frequency as measured at the monitor station and the mobile receiver serves to locate the latter on a particular member of the family of hyperbolas having foci at the known locations of the focal point transmitters. A second pair of transmitters provides a set of intersecting hyperbolas, and the phase comparison relative to the second set then locates the mobile receiver.

Research on interpretive methods has included theoretical investigations of the effects of vertical dikes and dipping beds on resistivity surveys, and special attention has been given to the principles underlying the application of telluric currents to prospecting. A close relation between theory and field application is maintained.

Seismic research has included detailed field studies to determine the behavior of extremely large patterns of shots and detectors. Play-back facilities at Montrouge consist of an Electro-Tech., Carter MT 4 group, and a Seismac installation with some added peripheral equipment. In order to get the most from reproducible recording, various record presentation methods are compared and an effort is made to see what benefits can be obtained by extreme filtering. Synthetic seismograms have been computed on their IBM 650 digital computer, including the effects of multiple reflections. In addition to its use in the routine calculations for Toran chains and for interpretation of electrical prospecting, the IBM 650 has been an invaluable aid in the calculations required in gravimetric and magnetic interpretation employing the methods of reduction to the pole.

Geophysical research at the Institut Francais du Petrole, Rueil Malmaison, Seine et Oise, France is one facet of a varied program covering exploration, oil recovery, and chemical processing. The newer buildings have been designed to blend with the original buildings of the estate on which the Institut is located, and fountains and gardens continue to add their charm to the surroundings.

Seismic research here also includes the production of synthetic seismograms, ${ }^{5}$ actually by three methods. In one case, the synthetic seismogram is obtained by inking one side of a continuous velocity trace to provide a variable-area film, feeding the photo-cell output through a circuit which takes the logarithm of the voltage, and then sending the signal through a HTL Time Domain Filter, in which the sensitivities of the heads have been adjusted to portray the desired basic reflection waveform. Synthetic seismograms including multiples are also obtained digitally on an IBM computer. The other method might be called "one-

${ }^{5}$ Editor's Note: See page 106, this issue. 
dimensional seismic modelling." An ultrasonic pulse of the desired waveform is sent down a brass rod in which the cross-sectional area has been machined in accordance with a continuous velocity log, and the reflection of this pulse then corresponds to the seismic field record, including all multiple reflections. Elastic waves in plastic plates have been used to study diffraction effects near faults and the properties of surface waves. Refraction interpretation is of interest.

The Institut is well equipped to make seismic record sections, having in operation a Carter play-back unit and a Seismac installation. They also have an experimental crew in the field. 